\title{
Performance and Industry Application Test of A High Power Access Point
}

\author{
Xu Liu, ${ }^{1, a}$, Da Zhang ${ }^{1, b}$, Xiao Lv ${ }^{1, c}$, Feng Jin ${ }^{1}$, ,XueSong Chen ${ }^{1}$ \\ ${ }^{1}$ Beijing General Research Institute of Mining \& Metallurgy, Beijing, China \\ aliuxu@bgrimm.com \\ bZhangda@bgrimm.com \\ Ivxiao@bgrimm.com
}

\begin{abstract}
Keywords: Access Point, Mining, Wireless Communication, Test
Abstract. This paper designs a high performance wireless communication access point based on 802.11n for mining for low communication speed and low transmission power in mine. The access point is composed of hardware layer and software layer. This access point supports double frequency at $2.4 \mathrm{GHz}$ and $5 \mathrm{GHz}$, and it can reach communication speed at $300 \mathrm{Mbps}$, which improved significantly performance of wireless communication for mining. We tested and optimized the access point in Lab. Finally we tested the access point in industry, the comprehensive test result showed that the access point can satisfy wireless communication requirements for mining.
\end{abstract}

\section{Introduction}

With the rapid development of computer and communication, a single cable communication is widely used in mining industry which becomes the performance bottleneck, and the high construction costs and fixed network topology has been difficult to adapt to the actual needs of safe and efficiency production in mine ${ }^{[1]}$. At present, the domestic research institutes have put forward a mixed network structure which mainly based on wired network and wireless network as a subsidiary, and which to a certain improved the extension and flexibility of the network. Industrial Ethernet has been widely used in mine enterprise, Wi-Fi wireless access technology access to markets. But the core technology remains basically in IEEE $802.11 \mathrm{~g}$ stage which only can provide the highest link data transmission at $54 \mathrm{Mbps}$. Also it is unable to complete HD video and real time control command transmission, which seriously limits overall performance of the network ${ }^{[2-4]}$.

This paper designs a high-performance wireless communication wireless access point based on $802.11 \mathrm{n}$ for mining. This access point supports double frequency at $2.4 \mathrm{GHz}$ and $5 \mathrm{GHz}$, and it can reach communication speed at $300 \mathrm{Mbps}$, which improved significantly performance of wireless communication for mining.

\section{Overall Design}

High power $\mathrm{Wi}-\mathrm{Fi}$ access point for mining is mainly used to build mining wireless communication network which holds data including HD video information, real-time voice information etc. multimedia data, environmental monitoring data and equipment control instruction. In those applications for underground, which require the following features:

(1) High speed of data transmission, which ensures data concurrent transmission in real time.

(2) Abundant physical interface, which realizes compatibility access for various of underground equipment.

(3) High transmitter power and rx sensitivity, which provides large enough wireless signal coverage and adapts to complex link environment .

(4) Steady operation, which ensures communication system reliability and robustness.

Based on the above requests, on the background for underground application, this paper used modular design to complete designing access point overall design. The hardware of the access point 
was composed of hardware layer and software layer . The overall framework is shown in Figure 1.The hardware mainly includes the core processor module, communication interface module and the wireless communication module which was composed of $5 \mathrm{GHz}$ RF module and $2.4 \mathrm{GHz}$ RF module. The software layer mainly includes Mips-Linux-2.6.31 kernel, hardware driver, IEEE 802.11n protocol stack, network protocol and application service etc..

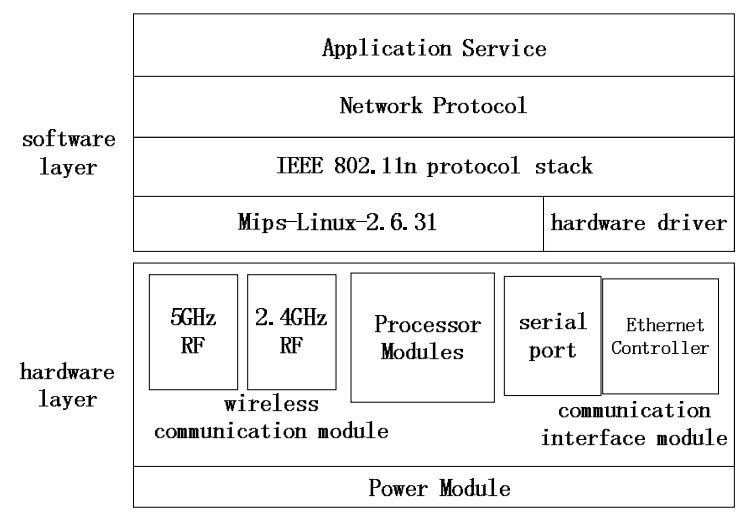

Fig. 1 Access Point general framework

\section{Performance Test}

To ensure access point performs well which can provide support the stable wireless communication for the industrial test. This paper tested the access point in many ways, including:

(1) RF indexes test.

(2) Ethernet and optical network communication test.

(3) Flow test.

\section{RF Indexes Test}

The wireless signal transmission falls into main direction: transmit and receive, the signal quality in every direction can affect the system communication effects. Litepoint IQ2010 is an $\mathrm{RF}$ equipment which integrates with transmitter and receiver. It has multiple concurrent compatible ability of wireless communication standard,so we chose it as access point test equipment to test the signal quality of transmit and receive.

We set the access point that debugged and optimized as test mode and loaded the test code, and then we use IQ2010 to calibrate and test. The test result is show as Figure 2.

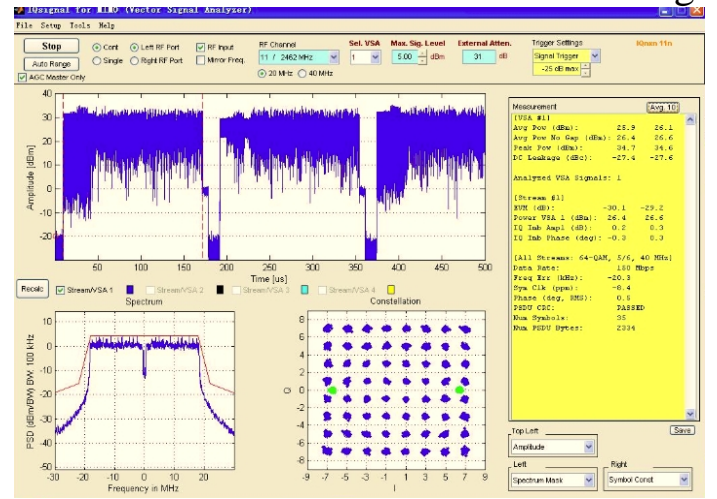

Fig. 2 The test result of signal quality of transmit and receive 


\section{Ethernet and optical network communication test}

The stable network communications is basis forthe system running stability. In real industrial environment, the reasons that the high rate packet loss and instability flow of the device case various network problems. To ensure good data communications channel, so we test the quality of network communication of the $1000 \mathrm{Mbps}$ Ethernet port and optical interface port of the communication system device. After we optimized above problems, and then got the following test results.

\section{Flow Test}

Table 1 Flow Test Result

\begin{tabular}{|l|c|c|l|c|}
\hline PacketsSent Per Second (PPS) & & & Throughput Test & \\
\hline Test Period & 60 & & Test Number & 1 \\
\hline Min Frame Size & Custom & & InitialS peed & Custom \\
\hline Max Frame Size & Custom & & Min Speed & Custom \\
\hline Frame Length Increment & Custom & & Max Speed & Custom \\
\hline Sending Mode & Bidirectional & & Speed Precision & Custom \\
\hline Frame Length & PacketsReceived & {$[1,1,2]->[1,1,3]($ PPS(MPS)) } & {$[1,1,3]-->[1,1,2]$ (PPS(MPS)) } & \\
\hline & & $1000 \mathrm{M}-1000 \mathrm{M}$ & $1000 \mathrm{M}--1000 \mathrm{M}$ & Total(PPS) \\
\hline 64 & $100 \%$ & 1488096 & 1488096 & 2976192 \\
\hline 128 & $100 \%$ & 844595 & 844595 & 1689190 \\
\hline 256 & $100 \%$ & 452899 & 452899 & 905798 \\
\hline 512 & $100 \%$ & 234963 & 234963 & 469926 \\
\hline 1024 & $100 \%$ & 119732 & 119732 & 239464 \\
\hline 1280 & $100 \%$ & 96154 & 96154 & 192308 \\
\hline 1518 & $100 \%$ & 81275 & 81275 & 162550 \\
\hline
\end{tabular}

The results showed that, the network flow transmission of access point can reache the industrial application level which provides high bandwidth and high quality in industrial field.

\section{Network Delay Test}

Table 2 Network Delay Test Result

\begin{tabular}{|l|l|c|l|c|}
\hline Time Delay Unit (us) & & & Throughput Test & \\
\hline & Test Period & & Test Number & 1 \\
\hline & Min Frame Size & & InitialS peed & Custom \\
\hline & Max Frame Size & & Min Speed & Custom \\
\hline & Frame Length Increment & Custom & Max Speed & Custom \\
\hline & Sending Mode & Bidirectional & Speed Precision & Custom \\
\hline & PacketsReceived & {$[1,1,2]-->[1,1,3](\mathrm{PPS}(\mathrm{MPS}))$} & {$[1,1,3]-->[1,1,2](\mathrm{PPS}(\mathrm{MPS}))$} & \\
\hline & & $1000 \mathrm{M}-1000 \mathrm{M}$ & $1000 \mathrm{M}--1000 \mathrm{M}$ & avagre \\
\hline & $100 \%$ & 1.570 & 1.540 & 1.555 \\
\hline & $100 \%$ & 1.700 & 1.630 & 1.655 \\
\hline 64 & $100 \%$ & 1.700 & 1.630 & 1.655 \\
\hline 128 & $100 \%$ & 1.700 & 1.630 & 1.655 \\
\hline 256 & $100 \%$ & 1.700 & 1.630 & 1.655 \\
\hline 512 & $100 \%$ & 1.700 & 1.630 & 1.655 \\
\hline 1024 & $100 \%$ & 1.720 & 1.650 & 1.685 \\
\hline 1280 & & & &
\end{tabular}

The results showed that, the network delay test of access point performed well. In the test of frame length of 1518 bytes, the average delay is only $11.598 \mu$ s, The access point can satisfy network delay in the industrial environment.

Table 3 Network Packet Loss Rate Test Result

\begin{tabular}{|c|c|c|l|c|}
\hline Network Packet Loss Rate Test & & Throughput Test & \\
\hline & Test Period & & Test Number & 1 \\
\hline & Min Frame Size & & Initials peed & Custom \\
\hline & Max Frame Size & & Min Speed & Custom \\
\hline & Frame Length Increment & Custom & Max Speed & Custom \\
\hline & Sending Mode & Bidirectional & Speed Precision & Custom \\
\hline & Rate (100\%) & $[1,1,2]->[1,1,3]$ (PPS(MPS) $)$ & {$[1,1,3]->[1,1,2]($ PPS(MPS)) } & \\
\hline Frame Length & & $1000 \mathrm{M}--1000 \mathrm{M}$ & $1000 \mathrm{M}-1000 \mathrm{M}$ & avagre \\
\hline & $100 \%$ & $0 \%$ & $0 \%$ & $0 \%$ \\
\hline 64 & $100 \%$ & $0 \%$ & $0 \%$ & $0 \%$ \\
\hline 128 & $100 \%$ & $0 \%$ & $0 \%$ & $0 \%$ \\
\hline 256 & $100 \%$ & $0 \%$ & $0 \%$ & $0 \%$ \\
\hline 512 & $100 \%$ & $0 \%$ & $0 \%$ & $0 \%$ \\
\hline 1024 & $100 \%$ & $0 \%$ & $0 \%$ & $0 \%$ \\
\hline 1280 & $100 \%$ & $0 \%$ & & \\
\hline 1518 & & & $0 \%$ & $0 \%$ \\
\hline
\end{tabular}




\section{Network Packet Loss Rate Test}

The results showed that the network packet loss rate of the access point is $0 \%$, and it can realize needs that high speed forward of communication in mining.

\section{Industry Test}

In this paper we chose ZhongJin LingNan Company Fankou lead-zinc Mine as test site.We construct the wireless network at $-240 \mathrm{~m}$ Ramp . By comprehensive consideration of trend, width, height and transmitted power of the access point, we layout the access point as the following Figure 3.

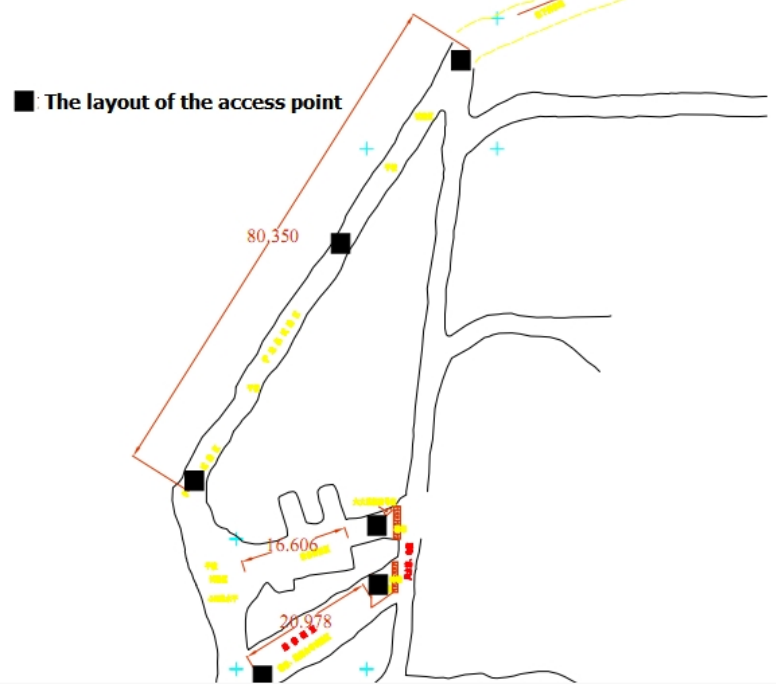

Fig. 3 The layout of the access point

In the experiments, the intelligent equipment transmit HD video, control command and sensor information to monitoring center by the wireless network. We test the network delay, band width , stability test of the wireless network.

The comprehensive test showed that,

(1) The speed of wired network reach 1000Mbps;

(2) Wireless link rate reach 300Mbps;

(3) Network packet loss rate $<0.003 \%$;

\section{Conclusions}

This paper introduces a high-performance wireless communication wireless access point based on 802.11n for mining. It presents the accs composition, which includes hardware composition and software design. Through the access point, which can make up the low communication speed and low transmission power in mining. It will have fine application prospects in the mining .

\section{Acknowledgements}

This research was supported by the National High Technology Research and Development Program of China (863 Program, Grant 2011AA060406) and International Cooperation Project (Grant 2011DFA71990) and the star of science and technology plan projects of Beijing(Z131101000413095) and National Natural Science Foundation (41301436)

\section{References}

[1]L. Nathawad,M. Zargari,H. Samavati,S. Mehta,A. Kheirkhahi. Solid-State Circuits Conference.ISSCC 2008. Digest of Technical Papers. IEEE International,2008: 358-619.

[2] L.Lin,N. Wongkomet,D. Yu,C.H. Lin. Solid-State Circuits Conference - Digest of Technical Papers. ISSCC 2009. IEEE International, 2009: 416 - 417,417a.

[3]D. Wan, Y. Du: Journal of Jiangnan University, Vol.6,No.5,Oct,2007: 542-546. 
[4]A. Balasubramanian,R. Mahajan,A .Venkataramani,B.N. Levine,J. Zahorjan. Acm Sigcomm Computer Communication Review, 2013, 38(4):427-438

[5] P. Mohanan,K. Vasudevan,G. Augustin,S.V. Shynu,C.K. Aanandan. Electronics Letters, 2006, 42(9):502-503.

[6] M.S. Ji,J.H. Han,W.K.Lee,W.G. Yang. IEEE Antennas \& Propagation Society International Symposium. (2006):3115-3118 